\title{
One thousand minimally invasive mitral valve operations: Early outcomes, late outcomes, and echocardiographic follow-up
}

\author{
R. Scott McClure, MD, SM, FRCSC, Leonidas V. Athanasopoulos, MD, PhD, Siobhan McGurk, MSc, \\ Michael J. Davidson, MD, Gregory S. Couper, MD, and Lawrence H. Cohn, MD
}

\begin{abstract}
Objective: The present study assessed the clinical and echocardiographic outcomes for 1000 patients undergoing minimally invasive mitral valve surgery.

Methods: The Brigham Cardiac Valve database was reviewed. From August 1996 to November 2011, 1000 patients had undergone minimally invasive mitral valve surgery (median follow-up, 7 years). Data on the surgical approach, complications, reoperations, and late survival were tabulated. Late echocardiographic data on the recurrence of mitral regurgitation after mitral repair in myxomatous disease were also collected. Survival, freedom from reoperation and recurrent mitral regurgitation (grade $\geq 3+$ ) were evaluated with life tables and Kaplan-Meier analyses.
\end{abstract}

\begin{abstract}
Results: The mean patient age was 57 years. Of the 1000 patients, $41 \%$ were women. Myxomatous degenerative disease was the predominant pathologic entity $(86 \%)$. A lower hemisternotomy was the predominant surgical approach $(75 \%)$. Mitral repair was performed in 923 patients and replacement in 77 . Eight operative deaths $(0.8 \%)$ occurred. A total of 44 patients with failed mitral repairs underwent reoperation, with 1 mitral valve replaced again on the same operative day for atrioventricular groove disruption. Nine failed repairs were repaired again (9/44 [20\%]). A total of 106 late deaths occurred. The overall survival at 15 years was $79 \% \pm 3 \%$. Freedom from reoperation at 15 years was $90 \% \pm 3 \%$ for repairs and $100 \%$ for replacements. Late echocardiograms were acquired for 615 of 815 eligible mitral repair patients with myxomatous disease $(75 \%)$. Freedom from recurrent mitral regurgitation (grade $\geq 3+$ ) at 1,5 , and 10 years was $99 \% \pm 1 \%, 87 \% \pm 2 \%$, and $69 \% \pm 4 \%$, respectively.
\end{abstract}

Conclusions: Minimally invasive mitral valve surgery is effective, with excellent late results. The durability of minimally invasive mitral valve repair compared favorably with conventional full sternotomy methods at late follow-up. (J Thorac Cardiovasc Surg 2013;145:1199-206)

Minimally invasive mitral valve surgery (mini-MVS) has been in use for 15 years. ${ }^{1,2}$ Built on the premise that less invasive surgery could expedite postoperative recovery owing to a reduction in surgical trauma with limited access techniques, resulting in reduced hospital stays, reduced hospital costs, improved cosmesis, and increased patient satisfaction, mini-MVS continues to be steadily adopted, with more and more advocates over time. ${ }^{3}$ At its inception, mini-MVS focused solely on smaller incisions, specifically the parasternal incision, and upper and lower hemisternotomy. Although the parasternal approach has fallen out of favor, the upper and lower hemisternotomy

From the Division of Cardiac Surgery, Brigham and Women's Hospital, Harvard Medical School, Boston, Mass.

Disclosures: Authors have nothing to disclose with regard to commercial support.

Read at the 38th Annual Meeting of The Western Thoracic Surgical Association,

Maui, Hawaii, June 27-30, 2012.

Received for publication June 27, 2012; revisions received Dec 8, 2012; accepted for publication Dec 18, 2012; available ahead of print Jan 25, 2013.

Address for reprints: Lawrence H. Cohn, MD, Division of Cardiac Surgery, Brigham and Women's Hospital, Harvard Medical School, Pbb J-101, 75 Francis St, Boston,

MA 02115 (E-mail: lcohn@ partners.org).

$0022-5223 / \$ 36.00$

Copyright () 2013 by The American Association for Thoracic Surgery

http://dx.doi.org/10.1016/j.jtcvs.2012.12.070 continue to be used by many, along with the now predominant approach at most centers, the small right anterior thoracotomy. In conjunction with these techniques, robotics and thoracoscopic instruments have gradually been introduced as adjuncts to further minimize the surgical trauma and have been championed at select cardiac centers. ${ }^{4,5} \mathrm{~A}$ multitude of studies have supported the safety of miniMVS in the immediate postoperative period, and a few select case series have shown excellent midterm outcomes with these techniques. ${ }^{1-9}$ However, data to assess the long-term outcomes with mini-MVS remain relatively sparse. Although establishing the safety of mini-MVS is pertinent, the true testament to its continued assimilation will be twofold: (1) to ensure that limited exposure with mini-MVS is not a direct cause for mitral valve replacement instead of mitral valve repair in patients for whom repair would clearly be the preferred therapy; and (2) to ensure the long-term mitral valve durability is on par with that achieved using conventional full sternotomy methods. If the perceived early benefits of mini-MVS are achieved at the expense of a greater propensity to replace complex regurgitant valves that would otherwise have been repaired, or at the expense of a greater reoperation rate secondary to subpar repair durability, the minimally invasive techniques 


\section{Abbreviations and Acronyms \\ mini-MVS $=$ minimally invasive mitral valve surgery \\ MR = mitral regurgitation}

will be counterproductive and arguably unwarranted. In an effort to critically appraise the long-term clinical and echocardiographic results with mini-MVS, we report on a consecutive series of 1000 patients who had undergone mitral valve surgery using minimally invasive techniques at the Brigham and Women's Hospital during a 15-year period.

\section{METHODS}

The present study was a retrospective, observational review of all patients who underwent a minimally invasive mitral valve procedure from July 1, 1996 to November 30, 2011. The institutional review board approved the study and waived patient consent.

The patient demographics, medical history, and operative and inhospital outcomes were collected at each patient's admission and at each consecutive follow-up visit. The data were coded according to the defined definitions of the Society of Thoracic Surgeons Adult Cardiac Database, version 2.52. Long-term mortality was documented through to December 31,2011 , with a review of the data collected from routine clinic followup visits, in addition to a query of the Social Security Death Index. Data on late reoperations or reintervention were similarly sought through routine clinic follow-up visits, periodic patient questionnaires, and contact with the primary care practitioners or referring cardiologists. The main outcomes of interest were early and late mortality, mitral valve-related reoperations, and the recurrence of moderate (grade $3+$ ) or severe (grade 4+) mitral regurgitation (MR). The postoperative in-hospital morbidity data for stroke, reoperation for bleeding, permanent pacemaker insertion, deep sternal wound infection, new-onset atrial fibrillation, renal failure, and packed red blood cell transfusions were also assessed.

Late follow-up echocardiograms (defined as $\geq 6$ months) were sought for all eligible patients with myxomatous degenerative disease who had undergone minimally invasive mitral valve repair. Late echocardiograms absent from the Brigham Cardiac Valve database were obtained from the primary care practitioners or referring cardiologists, when available. When assessing late MR recurrence, the echocardiogram on file that was farthest from the initial surgery or, in the case of reoperation, the echocardiogram immediately before that reoperation, was used. Recurrent MR was defined as moderate or severe on a 4-point scale $(1+$ [trace], $2+$ [mild], $3+$ [moderate], and 4+ [severe]). The exclusion criteria for recurrent MR analysis were patients who had undergone mitral valve replacement, patients who had undergone mitral valve repair for a nonmxyomatous etiology, patients with less than 6 months of follow-up because of early death or early reoperation, and patients with less than 6 months from surgery to the start of the study. Of the 923 patients who underwent mitral repair, 108 patients met the exclusion criteria, leaving 815 eligible patients. Of these 815 patients, a late follow-up echocardiogram was acquired in 615 (75\%).

\section{Surgical Techniques}

Patient selection was predicated by surgeon preference. Absolute contraindications to a minimally invasive approach included surgically significant coronary artery disease, chest wall deformities, and morbid obesity.

Surgical access was predominantly through a lower hemisternotomy. Our technique has been described previously. ${ }^{10}$ In brief, a 6-cm skin incision, 2 fingerbreadths distal to the manubrium, was extended distal to a level 2 fingerbreadths proximal to the xiphoid process. A hemisternotomy was performed from the xiphoid to the second intercostal space and extended into the second intercostal space on the right side using an oscillating saw. The ascending aorta was cannulated directly with percutaneous cannulation of the inferior vena cava by way of the femoral vein with the assistance of transesophageal echocardiography.

When using a right anterior thoracotomy, our approach was similar to that described by others, ${ }^{8}$ with slight modifications. Access was primarily through the fourth intercostal space by way of a 6-cm skin incision made either just below the nipple or within the inframammary crease for men and women, respectively. Cardiopulmonary bypass was initiated by way of the femoral artery and vein cannulation through a small transverse incision in the groin. An 8-mm tube graft was sewn to the femoral artery and attached to the arterial cannula, and the femoral vein was percutaneously accessed and guided up to the inferior vena cava-right atrial junction, again with transesophageal echocardiography guidance. Percutaneous cannulation of the superior vena cava was also instituted to maximize venous drainage. The aorta was crossclamped using the flexible Cosgrove aortic clamp (CareFusion V. Mueller, Waukegan, Ill) directly through the thoracotomy incision.

For both approaches, the lower hemisternotomy and right anterior thoracotomy, barring factors that would necessitate access to the right atrium (tricuspid valve pathologic features, patent foramen ovale), the mitral valve is preferentially approached through the interatrial groove. Moreover, undersize (16F-24F) vacuum-assisted cannulas are used with both techniques to optimize exposure and maintain venous drainage. Myocardial protection is primarily through antegrade cardioplegia for both techniques.

\section{Statistical Analysis}

All statistical analyses were performed with the assistance of a departmental statistician. Fisher's exact test was used to evaluate the dichotomous variables. $t$ Tests with Levine's homogeneity of variance or the MannWhitney $U$ test were used for continuous variables, as appropriate. Survival and freedom from recurrence of moderate or severe (grade $\geq 3+$ ) MR were evaluated with life tables and Kaplan-Meier analyses. Categorical and dichotomous variables are presented as numbers and percentiles. Continuous variables are presented as the mean \pm standard deviation (normally distributed) or median and interquartile range (non-normal distributions). All statistical analyses were performed using SPSS, version 13.0 (SPSS, Chicago, Ill).

\section{RESULTS}

A total of 3133-isolated mitral valve surgeries (defined as MVS without coronary artery bypass grafting) were performed from August 1, 1996 to November 30, 2011. Mitral valve reparative techniques were used in 2127 patients $(68 \%)$, and 1006 underwent mitral valve replacement $(32 \%)$. Of the patients from this cohort, 1000 (32\%) underwent MVS with a minimally invasive approach and were the focus of the present review. Minimally invasive mitral valve repair was the predominant treatment choice $(923 / 1000$ [92\%]), with a small percentage undergoing minimally invasive mitral valve replacement (77/1000 [8\%]). The median cardiopulmonary bypass time for the complete cohort was 118 minutes, and the median crossclamp time was 88 minutes. A single surgeon (L.H.C.) performed $77 \%$ of the minimally invasive cases.

The patient demographics are listed in Table 1 . The mean age was $57.2 \pm 13.4$ years, the mean ejection fraction was $62.5 \% \pm 8 \%$, and 408 of the patients were women $(41 \%)$. Myxomatous degenerative disease was the predominant 
TABLE 1. Baseline demographics $(n=1000)$

\begin{tabular}{|c|c|}
\hline Variable & Value \\
\hline \multicolumn{2}{|l|}{$\operatorname{Age}(y)$} \\
\hline Mean \pm standard deviation & $57 \pm 13$ \\
\hline Range & $17-90$ \\
\hline Women (n) & $408(41)$ \\
\hline \multicolumn{2}{|l|}{ NYHA functional class } \\
\hline Mean \pm standard deviation & $2.0 \pm 0.7$ \\
\hline Class III-IV (n) & $244(24)$ \\
\hline History of cerebrovascular accident (n) & $21(2)$ \\
\hline History of renal failure (n) & $10(1)$ \\
\hline Reoperation (n) & $12(1)$ \\
\hline Ejection fraction $(\%)$ & $62.5 \pm 8$ \\
\hline \multicolumn{2}{|l|}{ Mitral valve pathologic findings (n) } \\
\hline Mitral regurgitation & $957(96)$ \\
\hline Mitral stenosis & $10(1)$ \\
\hline Mitral regurgitation and mitral stenosis & $33(3)$ \\
\hline \multicolumn{2}{|l|}{ Mitral valve disease (n) } \\
\hline Myxomatous & $860(86)$ \\
\hline Rheumatic & $64(6)$ \\
\hline Dilatative cardiomyopathy & $36(4)$ \\
\hline Endocarditis & $31(3)$ \\
\hline Ischemic & $4(0.4)$ \\
\hline Mitral regurgitation grade $(0-4)$ & $3.80 \pm 0.4$ \\
\hline
\end{tabular}

pathologic entity ( $86 \%, 860$ patients). Surgical access was achieved through a lower hemisternotomy in 746 cases $(75 \%)$ and a right anterior thoracotomy in $43(4 \%)$. A parasternal incision was used in 204 patients $(20 \%)$ early in our minimally invasive experience, with an additional 5 patients undergoing upper hemisternotomy and 2 patients left anterior thoracotomy.

Specific to mitral valve repair, posterior leaflet resection with a modified leaflet advancement and insertion of an annuloplasty ring was the predominant reparative technique. Commissuroplasty, foldoplasty, and adjunct maneuvers such as edge-to-edge repair or an imbrication stitch were used, as indicated. The use of these techniques and other techniques, and their distributive use within the cohort, have been previously outlined. ${ }^{7}$ The use of these reparative techniques has remained relatively constant since last reviewed.

\section{Early Outcomes}

The postoperative mortality and morbidity were low. In-hospital mortality was $0.8 \%$ (8 patients), with a median length of hospital stay of 5 days. A total of 21 patients ( $2 \%$ ) incurred a permanent stroke, 19 patients $(2 \%)$ required reoperation for bleeding, 7 patients incurred new-onset postoperative renal failure $(0.7 \%), 6$ patients $(0.6 \%)$ required a permanent pacemaker, and 6 patients $(0.6 \%)$ developed a deep sternal wound infection. New-onset atrial fibrillation occurred in 201 patients $(20 \%)$. Only 297 patients $(30 \%)$ were transfused at least $1 \mathrm{U}$ of packed red blood cells. Of
746 patients, $6(1 \%)$ required conversion to full sternotomy. Access was through a lower hemisternotomy in each case; 2 cases were for an inability to defibrillate, 3 for poor exposure, and 1 for repair of an aortic dissection. Five notable, serious complications occurred. Early in our experience, 3 patients incurred aortic dissections related to cardioplegia needle placement. Each case was with a different surgical approach (parasternal incision in 1, right anterior thoracotomy in 1, and lower hemisternotomy in 1). The lower hemisternotomy was converted to full sternotomy; however, in the parasternal and thoracotomy cases, the aortic dissections were quite focal, enabling repair through the original surgical incision. One additional patient had a complicated course with biventricular failure occurring when coming off cardiopulmonary bypass, subsequent cardiogenic shock, placement of a biventricular assist device, and eventual heart transplantation. Finally, an elderly patient with a heavily calcified posterior annulus underwent mitral valve replacement and experienced an atrioventricular groove disruption on postoperative day 1 , necessitating emergent reoperation. All 5 of these patients survived to hospital discharge.

\section{Mitral Valve Replacement}

For the 77 mitral valve replacements, rheumatic valve disease $(\mathrm{n}=35,45 \%)$ and myxomatous degeneration $(\mathrm{n}=34$, $44 \%$ ) were the predominant pathologic entities. Endocarditis in 6 patients, a double orifice congenital defect in 1, and presumed radiation-induced valvular dysfunction in 1 patient completed the cohort. Of the 77 replacements, 6 were planned repairs that inevitably required intraoperative replacement $(8 \%)$. Of these 6 failed intraoperative repairs, 3 were complex regurgitant valves with myxomatous Barlow's type disease, 2 had excessive annular calcifications, and 1 case had protracted leaflets in the setting of previous radiation for lymphoma, as noted in this paragraph.

\section{Mitral Valve Repair}

Intraoperative success using minimally invasive mitral valve repair techniques was achieved in $98 \%$ of the cases. Of the 929 patients who underwent attempted mitral valve repair, 6 had an intraoperative failed repair, necessitating mitral valve replacement. For the remaining 923 patients with successful repair at surgery, 17 required intraoperative conversion to sternotomy $(\mathrm{n}=6)$ or reoperation within 6 months postoperatively $(n=11)$. Thus, of the 929 patients, $906(97.5 \%)$ did not require conversion or reoperation. At late follow-up, 44 mitral valve repairs had required a reoperative procedure. Repeat repair was accomplished in 9 $(20 \%)$, and $35(80 \%)$ necessitated replacement.

\section{Late Outcomes}

The survival curve for the complete 1000-patient cohort is shown in Figure 1. Follow-up for late survival was 


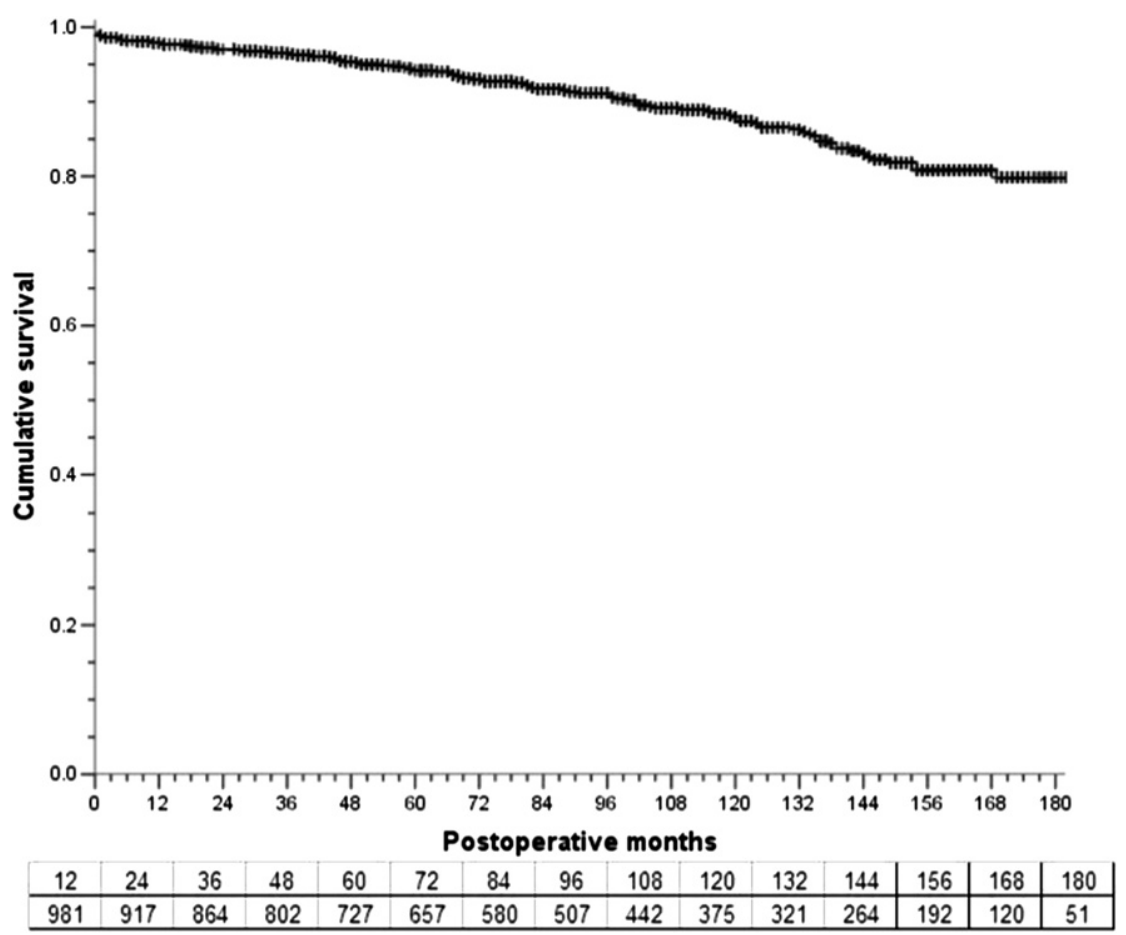

FIGURE 1. Survival for 1000 patients who had undergone minimally invasive mitral valve surgery at late follow-up. Tabular data provide number at risk in period ending on month noted. Estimates (mean \pm standard error) indicate 5-, 10-, and 15-year intervals.

$100 \%$ complete (median follow-up, 7.5 years; 7657 patientyears). Survival at 5,10 , and 15 years was $93 \% \pm 1 \%$, $86 \% \pm 1 \%$ and $79 \% \pm 3 \%$, respectively (median survival, 15 years; $95 \%$ confidence interval, 14.9-15.4). Freedom from reoperation was $100 \%$ for mitral valve replacement at late follow-up. For mitral valve repair, freedom from reoperation at 5, 10, and 15 years was $96 \% \pm 1 \%$, $95 \% \pm 1 \%$ and $90 \% \pm 3 \%$, respectively (Figure 2 ).

Long-term echocardiographic data were available for 815 patients who had undergone minimally invasive mitral valve repair for myxomatous degenerative disease. Of these, data extending 6 months or longer from surgery were available for 615 patients $(75.4 \%)$. A responder bias analysis comparing the cohort without follow-up with the study cohort showed the 2 groups to be substantially similar $(P \geq .2)$ in age, gender, New York Heart Association functional class distribution, ejection fraction, disease etiology, and surgical approach (data not shown).

The median interval to a postoperative echocardiographic study was 4.6 years (range, $0.5-13.4$ years), with a total of 3012 patient-years of acquired follow-up time. Freedom from recurrent MR for the entire cohort (defined as MR grade $\geq 3+$ ) at 1 year was $98.5 \% \pm 0.5 \%$ (n at risk $=589$ ), at 5 years was $87.3 \% \pm 1.7 \%$ (n at risk $=257$ ), and at 10 years was $68.8 \% \pm 4.1 \%$ (n at risk $=56$ ). Because it is well established that anterior leaflet and bileaflet pathologic features increase mitral valve repair complexity and can have a direct effect on late MR recurrence, we assessed the durability of mitral valve repair for anterior and bileaflet pathologic entities as a distinct group separate from patients with posterior leaflet pathologic features. ${ }^{11-13}$ The Kaplan-Meier analysis and freedom from recurrent MR for patients with posterior leaflet repair (436/ $615,70.8 \%)$ versus anterior or bilateral leaflet repair (179/ $615,29.1 \%$ ) are shown in Figure 3. Mitral valve repair durability was significantly less effective for patients with anterior or bileaflet repair than for posterior leaflet repair $(P=.017)$. Freedom from recurrent MR for posterior leaflet repair was $97 \% \pm 1 \%$ at 1 year, $88 \% \pm 2 \%$ at 5 years, and $76 \% \pm 4 \%$ at 10 years. In contrast, the 1-, 5-, and 10-year freedom from recurrent MR for anterior or bileaflet repair was $96 \% \pm 2 \%, 84 \% \pm 4 \%$, and $49 \% \pm 9 \%$, respectively. The repair durability was similar between the 2 groups up to the 7-year mark, at which point those with anterior leaflet and bileaflet pathologic features had a significantly greater precipitous increase in MR recurrence than patients with posterior leaflet repairs. Event-free survival (cumulative freedom from death, reoperation, and recurrent MR) for the myxomatous cohort (815 patients) who had undergone minimally invasive mitral repair is depicted in Figure 4. At 15 years, event-free survival was $77 \% \pm 4 \%$.

\section{DISCUSSION}

Mini-MVS, aside from patient satisfaction and aesthetics, has long been advocated for the potential benefits that it can provide to patients in the immediate 


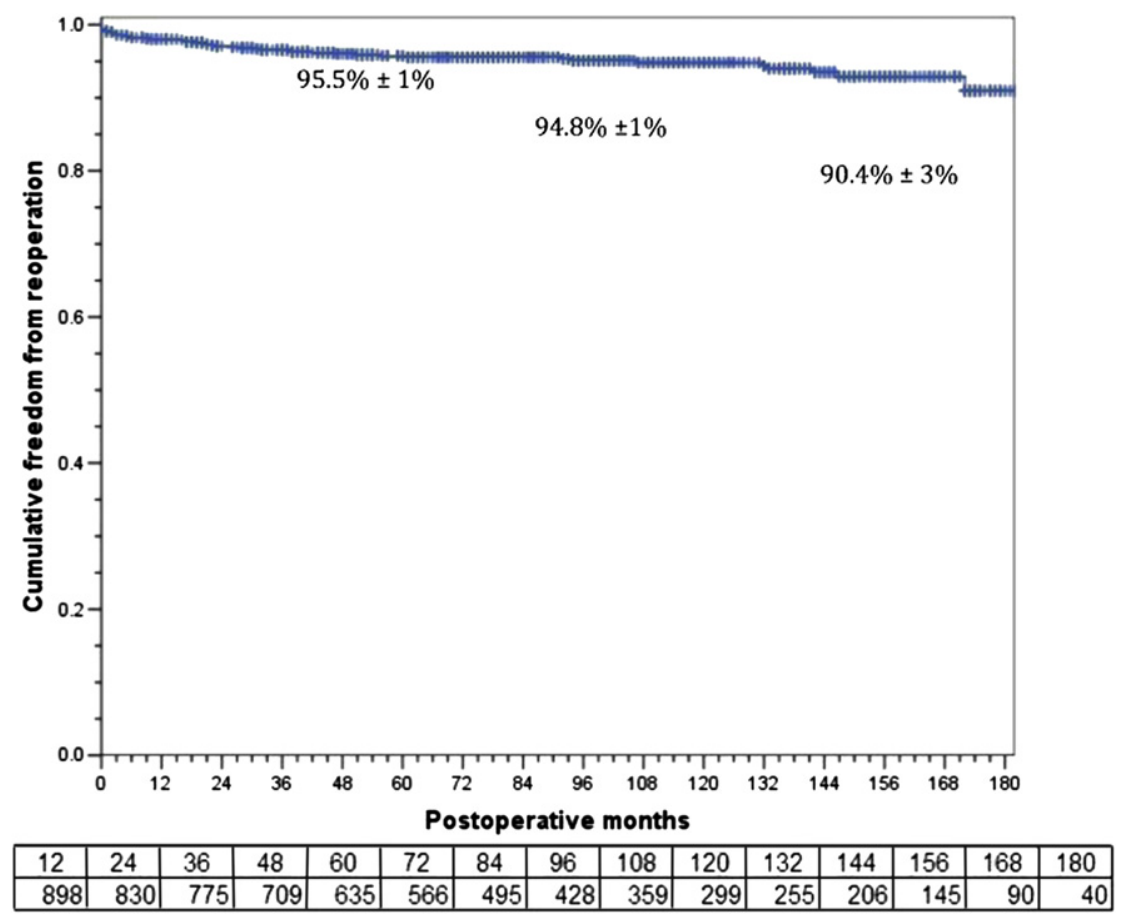

FIGURE 2. Freedom from reoperation for patients who had undergone minimally invasive mitral valve repair at late follow-up. Tabular data provide number at risk in period ending on month noted. Estimates (mean \pm standard error) indicate 5-, 10-, and 15-year intervals.

postoperative period. An exhaustive review of the most currently available evidence by Cheng and colleagues ${ }^{14}$ suggested mini-MVS to be associated with decreased bleeding, blood transfusions, atrial fibrillation, sternal wound infections, ventilation times, stay in the intensive care unit, and length of hospital stay and a more expeditious return to normal activity. Moreover, these benefits result without incurring any adverse effects in regard to valvular reintervention or survival at follow-up extending to 8 and 9 years, respectively, in select studies. ${ }^{14}$ Although these presumed benefits are compelling and support the continued use of minimal access techniques for mitral valve surgery, important unanswered questions remain. The assurance that these benefits can be achieved without influencing the intraoperative surgical preference toward replacement over repair and the assurance that these benefits can be achieved without jeopardizing the long-term rates of reoperation or mitral valve durability are unknowns that need additional study if mini-MVS is to continue to achieve more widespread acceptance. Clearly, mini-MVS comes with the adage that inherently less exposure will be available for the surgeon than that provided through a conventional full sternotomy. Thus, some have challenged the use of mini-MVS. ${ }^{15}$ It has been suggested (quite reasonably) that limited exposure could lead to surgical compromise, with the surgeon adapting their repair techniques to accommodate their limited space and inevitably accepting a lower quality repair or having a lower threshold for mitral valve replacement for valves that are truly repairable. ${ }^{15}$ Both are valid concerns.

Encouragingly, the present review of our initial 1000 minimally invasive mitral valve procedures with follow-up extending out 15 years does not substantiate either of these points of contention. Neither the intraoperative commitment to repair nor the long-term outcomes appeared to be negatively affected by the used of minimally invasive techniques.

The repair rate for the present series was exceedingly high at $97.5 \%$, with only 6 cases in which the initial attempt at repair ultimately required replacement. We assessed these findings more critically with a sensitivity analysis. For this, we assumed that all 34 myxomatous mitral valves that ultimately required mitral valve replacement could actually have been repaired and that the field of exposure was the deciding factor, rather than the complexity of the myxomatous pathologic findings. Given these assumptions, the repair rate for the entire cohort remained an impressive $94.6 \%$ (if 31 additional myxomatous valves had failed repair, the denominator would be $929+29=958$, for a mitral valve repair rate of $906 / 960=94.6 \%$ vs $906 /$ $929=97.5 \%$ ). With a mitral valve repair rate of $95 \%$ or greater, it is difficult to conceive that the use of a minimally invasive technique had much of an influence, if any, on the eventual intraoperative surgical therapy used. The decision to perform repair or replacement was more likely dictated by the valve pathologic features and the complexity of the disease process at intraoperative assessment. 


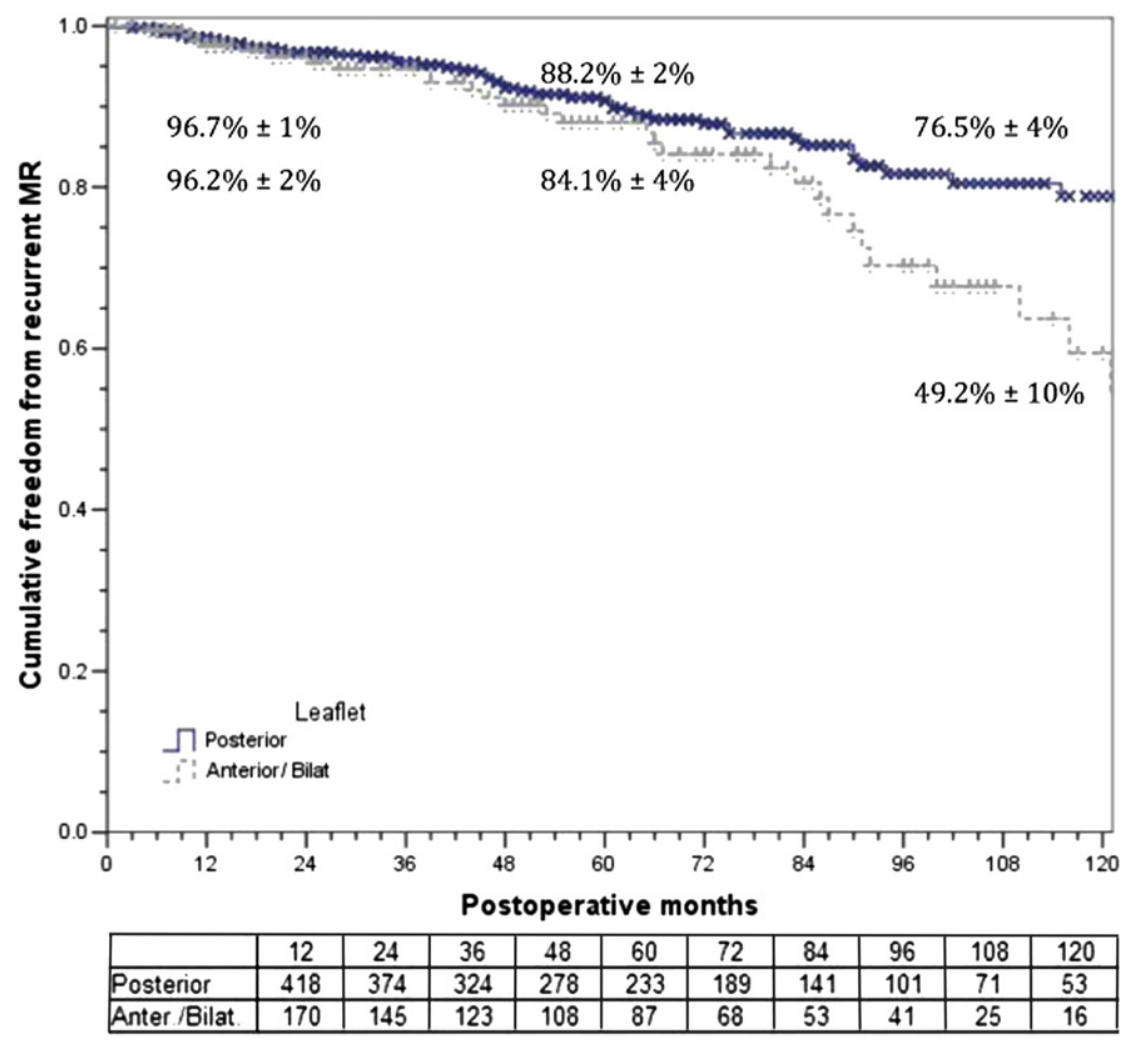

FIGURE 3. Freedom from recurrent (grade $\geq 3+$ ) mitral regurgitation $(M R)$ at late follow-up after minimally invasive mitral valve repair for myxomatous degenerative disease. Patients were stratified into 2 groups: patients with posterior leaflet pathologic features and patients with anterior or bileaflet pathologic features. Tabular data provide number at risk in period ending on month noted. Estimates (mean \pm standard error) indicate 1-, 5-, and 10-year intervals.

However, it should be emphasized that this is a selected group of patients and not every patient is suitable for mini-MVS. It is our belief that patient selection in the preoperative assessment is the foremost important predictor for success or failure with mini-MVS and that this assessment best dictates whether successful mitral repair is foreseeable. Very obese patients, an excessively deep thoracic cavity, and chest wall deformities can present formidable challenges when using minimally invasive techniques. We generally recommend against the use of mini-MVS for such patients.

In keeping with this, which highlights the notion that miniMVS does not necessarily have an influence on intraoperative surgical decision-making insofar as whether to repair or replace a particular valve, our long-term data also suggest that the clinical outcomes and mitral valve repair durability are not compromised by using mini-MVS techniques.

The freedom from recurrent MR (grade $\geq 3+$ ) for myxomatous disease of the posterior leaflet was $76 \% \pm 4 \%$ at 10 years in our series. This was significantly better than the $49 \% \pm 9 \%$ freedom from recurrent MR achieved for the anterior/bileaflet group during the same period. Both of these results are in line with that achieved by other groups assessing the recurrence of late MR and mitral valve repair durability for myxomatous disease using conventional full-sternotomy methods. Specifically David and colleagues, ${ }^{11}$ in a cohort of 701 patients with myxomatous disease who underwent a full sternotomy approach, noted a late rate of recurrent $\mathrm{MR}$ (grade $\geq 3+$ ) to be $80 \% \pm 4 \%$ for posterior pathologic features, $67 \% \pm 6 \%$ for anterior pathologic features, and $65 \% \pm 8 \%$ for bileaflet pathologic features at 12 years of follow-up. Admittedly, direct comparisons across these observational studies could be misleading; these were different patient populations with differing follow-up methods. Intraobserver variability among echocardiographers could also play a role. Also, the diminishing return on outcome reliability as the number of patients at risk continues to decrease with time also needs to be considered. Still, even when factoring in such shortcomings, it is more likely that these studies are reasonably comparable than drastically dissimilar. The Cleveland Clinic has also published data to support the long-term durability of mitral valve repair with minimally invasive techniques. In a recent review of more than 3000 patients with mitral valve repair for myxomatous disease of the posterior leaflet, freedom from recurrence of MR was as great as $89 \%$ at 10 years for the cohort, and $60 \%$ of the cases were performed using minimally invasive access (upper hemisternotomy). ${ }^{16}$ Taken together, these 3 studies suggest that valve durability can be maintained long-term with either 


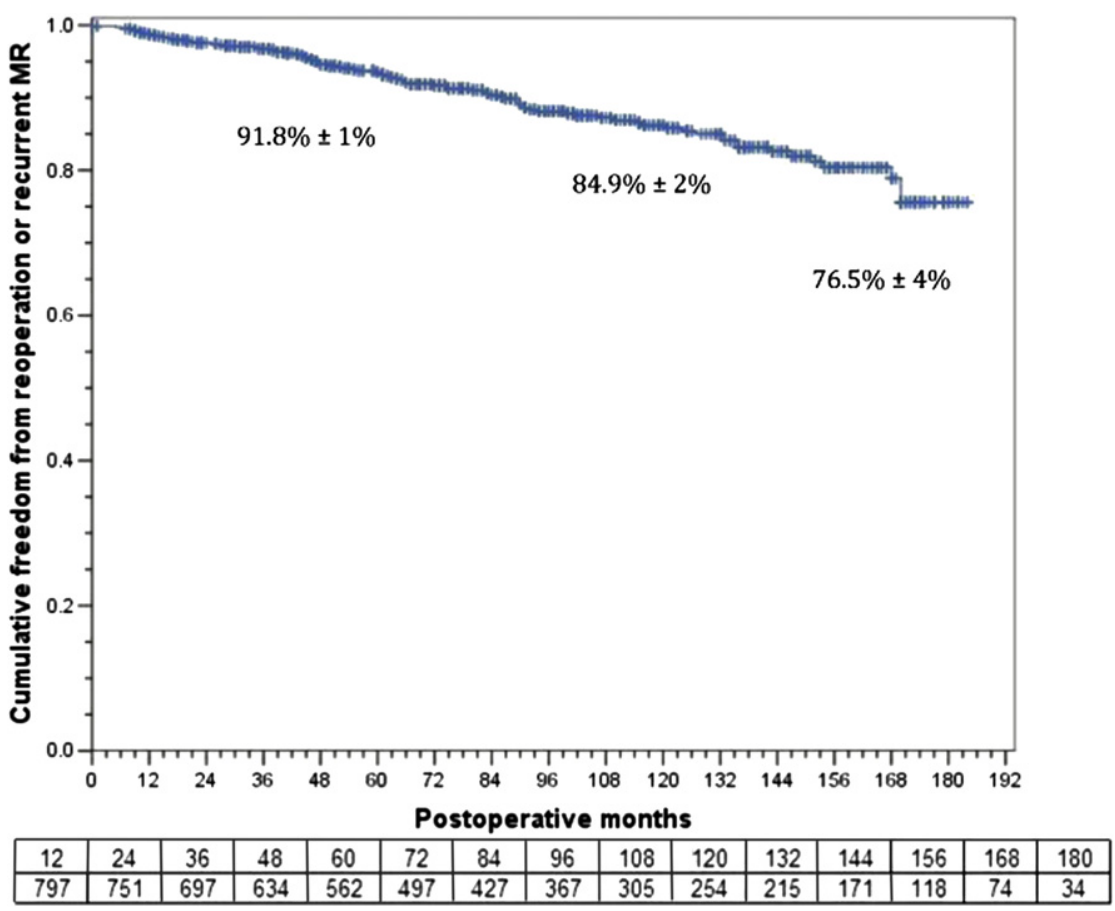

FIGURE 4. Event-free survival at late follow-up in patients who underwent minimally invasive mitral valve repair for myxomatous degenerative disease. Tabular data provide number at risk in period ending on month noted Estimates (mean \pm standard error) indicate 5-, 10-, and 15-year intervals. MR, Mitral regurgitation.

conventional full sternotomy or minimally invasive techniques without jeopardizing the quality of the repair.

However, despite the potential benefits of mini-MVS and the results of our study to suggest the long-term durability to be maintained using these techniques, mini-MVS has potential drawbacks that still need to be addressed. An increased risk of stroke, aortic dissection, and groin complications and increased crossclamp and cardiopulmonary times have all been mentioned as being of greater risk when performing mini-MVS versus conventional techniques. ${ }^{3,14}$ In our particular series, such complications have remained low.

\section{Study Limitations}

The present study was subject to the limitations inherent to a single-center, retrospective, study design. The decision to use minimally invasive surgery was surgeon specific, and the outcomes at our center might not be generalizable to other populations.

Although we retained as many subjects as possible for our echocardiographic analyses, echocardiographic follow-up data were missing for $25 \%$ of eligible subjects. Although our responder bias analysis would suggest similarities between the cohort analyzed and those who were lost to follow-up, to the degree that these additional results would have altered the final analysis could not be known with certainty. Because it is more likely that patients with a functioning mitral valve repair who remain asymptomatic would be less likely to present for a follow-up echocardiogram versus their symptomatic counterparts, it was our assumption that in this regard our analysis overestimated the recurrence of significant (grade $\geq 3+$ ) MR, but this is purely speculative.

Furthermore, the present study might not have accurately estimated the incidence of MR recurrence, because followup echocardiographic studies are not routine. It is possible that the follow-up was biased toward subjects with symptoms of MR or a high clinical suspicion for recurrence, which would, again, have overestimated long-term MR recurrence.

However, in contrast, by assessing the long-term recurrence of MR by relying on only 2 points in time (preoperative echocardiographic MR vs the latest echocardiogram on file with MR), our methods would tend to underestimate the cohorts' incidence of long-term MR recurrence, because patients were credited with the maximum time until the return of pathology (grade $\geq 3+\mathrm{MR}$ ). To better delineate the exact timing of MR over time, serial echocardiograms and statistical methods beyond the scope of the present study should be used. Still, because we are generally more interested in the interval to clinical reintervention, instead of the exact time at which grade $\geq 3+$ MR has occurred, we believe our methods were a rather reasonable proxy.

We our confident in our long-term mortality data; however, we acknowledge that the Social Security Death Index was only reliable to November 1, 2011, at which point access to this biomedical resource for vitality statistics was terminated. With our end date for follow-up at December 31,2011 , it is plausible that a long-term mortality event 
could have been missed within this 2-month discrepancy $(<5$, given our mortality rate estimations by life analyses), although it is our contention that if a few events were missed, they were highly unlikely to have had a substantial effect on our observations or conclusions. With our annual clinic and questionnaire follow-up protocols, the likelihood of having missed an event within this period was small (the total number of deaths in 2011 was 1 , and the year with the greatest mortality had $<2 / \mathrm{mo}$ ) and unlikely to have any effect on our overall analysis.

We are also confident in our long-term reintervention follow-up data, which were exhaustively accrued from many sources, as discussed in the "Methods" section. Despite our exhaustive efforts, it is always plausible within the framework of observational data collection that a reintervention occurred at another institution that was not accounted for in our follow-up data.

\section{CONCLUSIONS}

Mini-MVS is effective, with excellent late results. The durability of mini-mitral repair compared favorably with that after conventional full sternotomy methods at late follow-up.

\section{References}

1. Cohn LH, Adams DH, Couper GS, Bichell DP, Rosborough DM, Sears SP, et al. Minimally invasive cardiac valve surgery improves patient satisfaction while reducing costs of cardiac valve replacement and repair. Ann Surg. 1997;226:421-6.

2. Cosgrove DM, Sabik JF, Navia JL. Minimally invasive valve operations. Ann Thorac Surg. 1998;65:1535-8.

3. Gammie JS, Zhao Y, Peterson ED, O'Brien SM, Rankin JS, Griffith BP. Less-invasive mitral valve operations: trends and outcomes from the Society of Thoracic Surgeons Adult Cardiac Surgery Database. Ann Thorac Surg. 2010;90:1401-8.
4. Chitwood WR Jr, Rodriquez E, Chu MW, Hassan A, Ferguson TB, Vos PW, et al. Robotic mitral valve repairs in 300 patients: a single-center experience. J Thorac Cardiovasc Surg. 2008;136:436-41

5. Casselman FP, Van Slycke S, Wellens F, De Geest R, Degrieck I, Van Praet F, et al. Mitral valve surgery can now routinely be performed endoscopically. Circulation. 2003;108(Suppl 1):1148-54.

6. Modi P, Hassan A, Chitwood WR Jr. Minimally invasive mitral valve surgery: a systematic review and meta-analysis. Eur J Cardiothorac Surg. 2008;34: 943-52.

7. McClure RS, Cohn LH, Wiegerinck E, Couper GS, Aranki SF, Bolman RM III, et al. Early and late outcomes in minimally invasive mitral valve repair: an eleven-year experience in 707 patients. J Thorac Cardiovasc Surg. 2009;137: $70-5$.

8. Seeburger J, Borger MA, Falk V, Kuntze T, Czesla M, Walther T, et al. Minimal access mitral valve repair for mitral regurgitation: results of 1339 consecutive patients. Eur J Cardiothorac Surg. 2008;34:760-5.

9. Galloway AC, Schwartz CF, Ribakove GH, Crooke GA, Gogoladze G, Ursomanno P, et al. A decade of minimally invasive mitral repair: long-term outcomes. Ann Thorac Surg. 2009;88:1180-4.

10. Mihaljevic T, Cohn LH, Unic D, Aranki SA, Couper GS, Byrne JG. One thousand minimally invasive valve operations: early and late results. Ann Surg. 2004;240: 529-34.

11. David TE, Ivanov J, Armstrong S, Christie D, Rakowski H. A comparison of outcomes of mitral valve disease with posterior, anterior and bileaflet prolapse. J Thorac Cardiovasc Surg. 2005;130:1242-8.

12. Gillinov AM, Blackstone EH, Alaulaqi A, Sabik JF III, Mihaljevic T, Svensson LG, et al. Outcomes after repair of the anterior mitral leaflet for degenerative disease. Ann Thorac Surg. 2008;86:708-17.

13. Dibardino DJ, El Bardissi AW, McClure RS, Razo-Vasquez OA, Kelly NE, Cohn LH. Four decades of experience with mitral valve repair: analysis of differential indications, technical evolution, and long-term outcome. J Thorac Cardiovasc Surg. 2010;139:76-82.

14. Cheng DCH, Martin J, Lal A, Diegeler A, Follinguet TA, Nifong LW, et al. Minimally invasive versus conventional open mitral valve surgery. Innovations. 2011; 6:84-103.

15. Anyanwu AC, Adams DH. Should complex mitral valve repair be routinely performed using a minimally invasive approach? Curr Opin Cardiol. 2012;27: 118-24.

16. Johnston DR, Gillinov MD, Blackstone EH, Griffin B, Stewart W, Sabik JF III, et al. Surgical repair of posterior mitral valve prolapse: implications for guidelines and percutaneous repair. Ann Thorac Surg. 2010;89:1385-94. 